\title{
Adverse Events among Winnipeg Home Care Clients
}

\author{
$\overline{\text { Keir G. Johnson }}$
}

\begin{abstract}
Patient safety research has focused almost exclusively on hospitals, with few studies investigating the safety of other healthcare sectors, including home care. Before measuring patient safety in home care, this study first sought to translate hospital-focused patient safety definitions and concepts to home care. A contextappropriate approach to measuring adverse events (AEs) in home care was developed using chart reviews prompted by a mixed screening process. These methods were then applied to measure the incidence, type, severity, cause, preventability and ameliorability of AEs among Winnipeg Home Care clients.
\end{abstract}

D

atient safety has recently received considerable attention, with prominent studies in Canada (Baker et al. 2004), the United States (Brennan et al. 2001; Thomas et al. 2000) and other countries examining this critical healthcare issue. Baker et al. (2004) found that $7.5 \%$ of hospital admissions in Canada resulted in an adverse events (AE) - defined as unintended injuries or complications resulting in death, disability or prolonged hospital stay that arise from healthcare management and not the patient's underlying condition. These studies capture only part of the picture, however, as patient safety research has been limited almost exclusively to the hospital sector, with little research in other care settings, including home care.
Home care is an integral and growing component of Manitoba's healthcare system. Approximately 16\% of seniors in Winnipeg use these services (Roos et al. 1999). The limited patient safety research in home care is focused primarily on adverse drug events (Ellenbeker et al. 2004; Gray et al. 1999) and potentially inappropriate medication use (Fialová et al. 2005; Golden et al. 1999; Meredith et al. 2001), with a lesser focus on other areas such as rehospitalization (Madigan et al. 2001) and operational failures (Bruno and Ahrens 2005). The last study examined errors rather than AEs and found that errors are caused not only by home care providers, but also by other healthcare providers, clients themselves and their informal caregivers. One study that examined AEs broadly in home care reported potential events, and might be more accurately interpreted as quality indicators (Madigan and Tullai-McGuinness 2004).

This study sought to fill this gap in research about AEs experienced by home care clients by translating hospital-centred patient safety concepts to the home care context, developing a method to measure AEs in home care and applying this method to measure the annual incidence, type, harm, cause, preventability and ameliorability of AEs among home care clients of the Winnipeg Regional Health Authority (WRHA). Ameliorability is a relatively new concept to $\mathrm{AE}$ research, accounting for events that could not have been prevented, but where the harm or impact could have been reduced if a different approach had been taken (Forster et al. 2003; Forster et al. 2004). 


\section{Study Setting}

The WRHA is responsible for healthcare service delivery to more than 700,000 people living in Winnipeg and elsewhere in Manitoba. As part of the WRHA portfolio, the Home Care program is provincially mandated and seeks to ensure safe community living and promote independence while avoiding or delaying institutionalization. The program facilitates earlier hospital discharges for patients with short-term post-acute-care needs; however, the large majority of clients have long-term needs, and the program helps them remain at home for as long as possible.

\section{Development of Definitions}

Common patient safety concepts were developed in the hospital environment. Their transferability to home care needs to be reexamined, because care provision is quite different than institutionalized care. Delivering care in the home is less controlled than in hospitals, delivered in varying unfamiliar environments and relies heavily on client choice, family action and health and social services delivered by other professionals.

Two consultation sessions were conducted with case managers, supervisors of direct service staff, team managers, specialists, quality managers and directors, to translate the concepts of harm and cause and to generate a home-care-specific definition of "adverse event." For this study, an adverse event is defined as "any harm to the client that negatively affects their overall health and/or functioning and is the result of care actions and/or inactions rather than the client's underlying condition." Harm can include physical and mental injuries, emotional distress, unneeded or unplanned hospitalizations, premature or inappropriate nursing home placement, and death. Result of care, or cause, includes a number of different providers, including the WRHA Home Care program (coordinators, direct service staff, "systems"), other healthcare providers, clients (self-care) and informal caregivers. Because clients' care is fragmented among several providers, this study considered AEs caused by any provider, not only home care providers.

\section{Methods}

Patient safety studies have used a variety of retrospective and prospective methods. This study used retrospective chart reviews prompted by a mixed screening process.

In retrospective studies, the availability of comprehensive clinical and functional data is critical. The WRHA Home Care program uses a comprehensive electronic record that includes client demographic information, case manager progress notes, assessments and care plans. The program uses the internationally developed Minimum Data Set for Home Care (MDS-HC) assessment tool (Landi et al. 2000; Morris et al. 1997). The care plan component includes home care services and the contributions of other healthcare providers, clients and informal caregivers. While the home care client file is not a system-wide electronic health record, it contains comprehensive information about clients' health and functioning that was used to identify AEs.

\section{Study Sample}

The study focus was on long-term clients, who account for the majority of clients in the Home Care program. There were 14,624 such clients in WRHA Home Care during 2004 (based on program statistics provided by WRHA). A random sample of 400 client files opened in February and March of 2004 was extracted from the electronic client file database. Clients were excluded if they were discharged within 30 days of intake, did not have a completed assessment, or were identified as palliative.

Table 1. Summary of screening protocol

\begin{tabular}{|c|c|}
\hline Screening Protocols & Sample Search Criteria \\
\hline Fall & $\begin{array}{l}\text { MDS-HC: At least } 1 \text { fall in last } 90 \text { days } \\
\text { Keyword: "fall" or "fell" } \\
\text { Occurrence Report: } 4.2 \text { Falls }\end{array}$ \\
\hline Injuries/breaks/fractures & $\begin{array}{l}\text { MDS-HC: Presence of fractures or other } \\
\text { injuries } \\
\text { Keyword: "injure" }\end{array}$ \\
\hline Skin problems or ulcers & $\begin{array}{l}\text { MDS-HC: Presence of pressure ulcer } \\
\text { Keyword: "ulcer" or "sore" }\end{array}$ \\
\hline Infections & $\begin{array}{l}\text { MDS-HC: Urinary tract infection and use of } \\
\text { indwelling catheter } \\
\text { Keyword: "infection" }\end{array}$ \\
\hline Medication-related events & $\begin{array}{l}\text { Keyword: "reaction" or "overdose" } \\
\text { Potentially inappropriate medication search } \\
\text { Occurrence Report: } 4.1 \text { Medications }\end{array}$ \\
\hline Hospitalization & $\begin{array}{l}\text { MDS-HC: At least } 1 \text { overnight hospital stay, } \\
\text { visit to the ER or emergent care in last } 90 \\
\text { days } \\
\text { Discharge: Hospitalized } \\
\text { Keyword: "hospital" or names of hospitals } \\
\text { in Winnipeg }\end{array}$ \\
\hline Nursing Home Placement & $\begin{array}{l}\text { Discharge: Placed in nursing home } \\
\text { Keyword: "panel" or "nursing home" or } \\
\text { "personal care home" }\end{array}$ \\
\hline Death & $\begin{array}{l}\text { Discharge: Deceased } \\
\text { Keyword: "death" or "died" }\end{array}$ \\
\hline \multicolumn{2}{|c|}{$\begin{array}{l}\text { Screening Types: } \\
\text { MDS-HC (Minimum Data Set for Home Care): Standardized assessment } \\
\text { tool used by WRHA Home Care. } \\
\text { Discharge: Discharge records including reason for discharge. } \\
\text { Keyword: Search for list of keywords in clients' progress notes. } \\
\text { Potentially Inappropriate Medication Search: Medications identified as } \\
\text { potentially inappropriate for seniors, as defined by expert panels. } \\
\text { Occurrence Report: Voluntarily reported incident report. }\end{array}$} \\
\hline
\end{tabular}




\section{Screening}

Screening protocols were developed to identify a variety of types of AEs clients might experience or the harm AEs could cause (Table 1). Screening was limited to the year following intake, as this study sought to measure the annual incidence of AEs.

The screening process had four components. First, clients' MDS-HC assessments and their discharge records were searched for items identifying possible AEs. Second, clients' progress notes were searched for keywords or phrases, developed by adapting lists from other studies (Forster et al. 2005; Murff et al. 2003) and findings from the consultation sessions. The search was case-insensitive and looked for matches that contained roots of keywords to ensure both singular and plural forms were included (e.g. a search for "fall" could yield matches for "falls" and "fallen") as well as common synonyms (e.g., "pressure ulcer" and "bed sore").

The third screening process involved searching assessment records and medication notepads for any potentially inappropriate medications, as defined by expert panels (Beers 1997; Fick et al. 2003; McLeod et al. 1997) using Fialová et al.'s (2005) search criteria. While the names of medications, dosage, route and frequency are noted in client files, Drug Identification Numbers are not consistently recorded. Consequently, this screen was limited to searching for the full names and key name fragments of potentially inappropriate medications, which could exclude some matches due to incorrect spelling. This search was limited to clients aged 65 and older, expected to account for most of the sample.
Table 2. Rating scales for judging adverse event and preventability/ameliorability

\begin{tabular}{|l|l|}
\hline Adverse Event Rating Scale & Preventability/Ameliorability Rating Scale \\
\hline 0 No event occurred & \\
\hline 1 Virtually no evidence event caused by care & 1 Virtually no evidence of preventability/ameliorability \\
\hline $\begin{array}{l}2 \text { Slight to modest evidence event caused by } \\
\text { care }\end{array}$ & $\begin{array}{l}2 \text { Slight to modest evidence of preventability/amelio- } \\
\text { rability }\end{array}$ \\
\hline $\begin{array}{l}3 \text { Not likely event caused by care } \\
\text { (less than 50-50, but close call) }\end{array}$ & $\begin{array}{l}3 \text { Not likely event could have been prevented/ameliorated } \\
\text { (less than 50-50, but close call) }\end{array}$ \\
\hline $\begin{array}{l}4 \text { More likely event caused by care (more than } \\
50-50, \text { but close call) }\end{array}$ & $\begin{array}{l}4 \text { More likely event could have been prevented/ } \\
\text { ameliorated (more than 50-50, but close call) }\end{array}$ \\
\hline $\begin{array}{l}5 \text { Moderate to strong evidence event caused } \\
\text { by care }\end{array}$ & 5 Strong evidence of preventability/ameliorability \\
\hline 6 Virtually certain evidence event caused by care & 6 Virtually certain evidence of preventability/ameliorability \\
\hline $\begin{array}{l}\text { Rating scales based on those used in existing hospital and post-discharge studies (Baker et al. 2004; Forster } \\
\text { et al. 2003; Forster et al. 2004). }\end{array}$ \\
\hline
\end{tabular}

Figure 1. Data collection and review methods with client counts

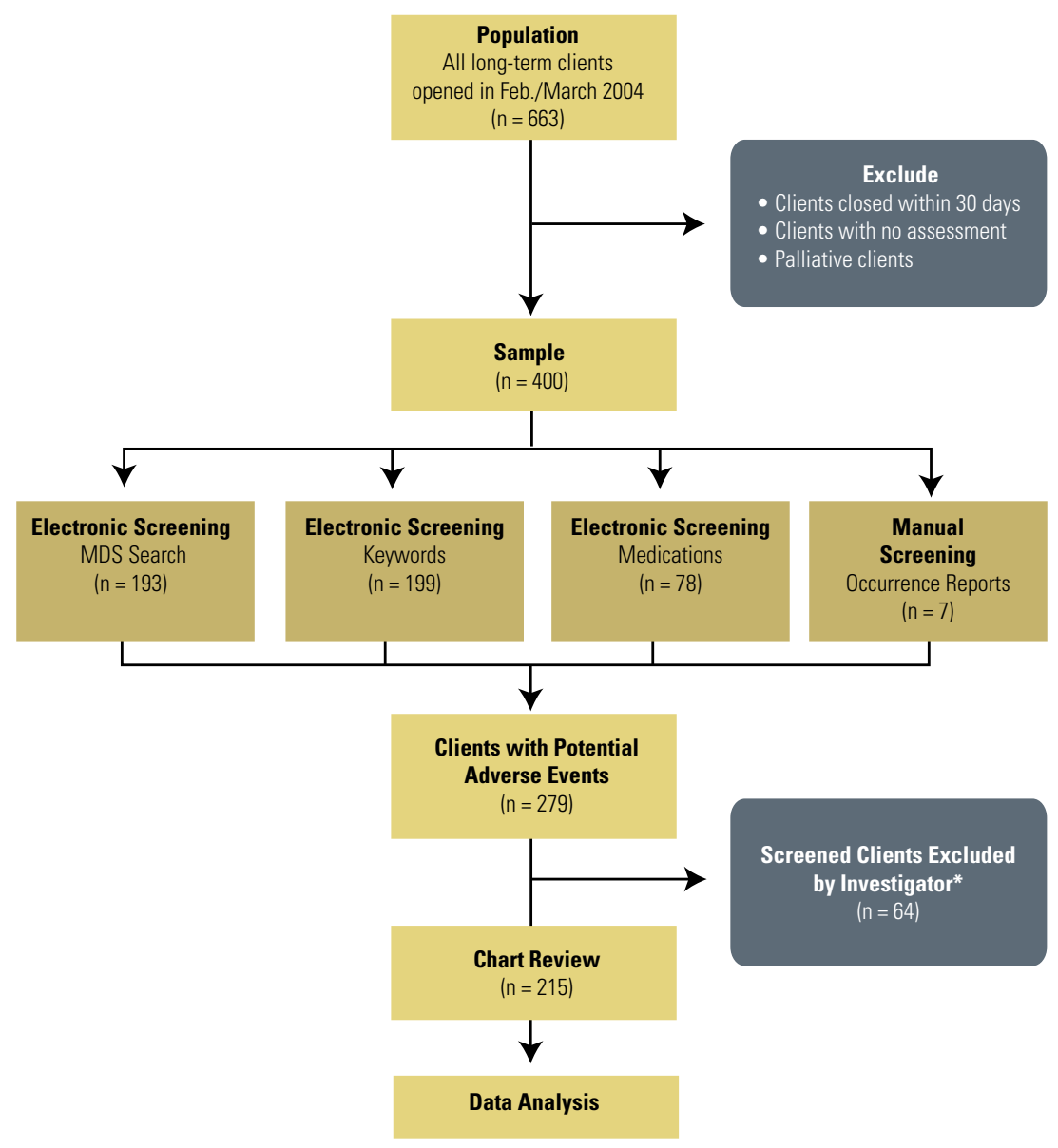

\footnotetext{
*Some screened-in clients were excluded because they clearly hadn't had an adverse event (i.e., the word "fall" screened the client in, but the word was used as "will follow-up in the fall") or potential event happened before client was open to home care.
} 
The Micromedex database was cross-referenced for all Canadian and American listings for noted medications.

The final screening approach searched WRHA occurrence (incident) reports. Occurrences are reported voluntarily by staff and filed centrally. An administrative assistant manually searched for occurrence reports on file for the study sample.

\section{Chart Reviewing}

Four trained chart reviewers reviewed charts for all clients who screened positive. Three of the reviewers were Home Care Specialists and one was a Team Manager; each had worked in home care for at least 15 years. Three of the reviewers were social workers and one a nurse. Chart reviewers attended a half-day training session to ensure they were familiar with the concepts used in this study, the screening process and chart reviews.

For all clients flagged during the screening stage, chart reviewers judged whether an $\mathrm{AE}$ had occurred. If an $\mathrm{AE}$ occurred, chart reviewers indicated the date, type of $\mathrm{AE}$, level of harm or impact to the client, and cause, and rated whether the $\mathrm{AE}$ could have been prevented or ameliorated. Scales for judging the likelihood an AE occurred and preventability/ameliorability were adapted from other studies (Table 2 provides sample rating scales). For clients judged to have had a preventable or ameliorable $\mathrm{AE}$, reviewers explained how the event could have been prevented or ameliorated. Reviewers also rated the adequacy of client records using an existing scale (Woloshynowych et al. 2003).

Chart review data were entered into an anonymized electronic database twice for accuracy. Scores for judging whether an $\mathrm{AE}$ had occurred and for preventability and ameliorability were dichotomized, with scores of four or more (at least 50\% certainty) indicating the presence of an $\mathrm{AE}$ or that the $\mathrm{AE}$ was preventable or ameliorable. Steps in the study methods are illustrated in Figure 1, along with the results for each step.

Approval was obtained from the University of Manitoba Joint-Faculty Research Ethics Board and the WRHA Research Review Committee.

\section{Results}

Characteristics of the study sample were compared with those of the home care population (Winnipeg Regional Health Authority 2005). The sample and population were similar in age and gender distributions, functional impairment and medication usage (Table 3 ), though the sample is slightly younger and less impaired, and took fewer medications.

From the sample, 279 clients (69.8\%) were flagged during the screening process (Figure 1). Several clients were identified by more than one method (Figure 2). Sixty-four of the screenedin clients were excluded during a brief manual review of the screens. Clients were only excluded if the context of a keyword match was clearly inappropriate (e.g., searching for the word
Table 3. Demographic and functional characteristics of sample and population

\begin{tabular}{|c|c|c|}
\hline & $\begin{array}{l}\text { Study Sample } \\
(n=400)\end{array}$ & $\begin{array}{l}\text { Home Care } \\
\text { Population* } \\
(n=10,991)\end{array}$ \\
\hline \multicolumn{3}{|l|}{ Age } \\
\hline Mean (SD, range) & $75.4(12.8,26-96)$ & $77.5(13.8,15-104)$ \\
\hline$\geq 65$ years & $81.5 \%$ & $87.8 \%$ \\
\hline$\geq 85$ years & $23.8 \%$ & $33.5 \%$ \\
\hline \multicolumn{3}{|l|}{ Gender } \\
\hline Female & $65.7 \%$ & $70.9 \%$ \\
\hline \multicolumn{2}{|c|}{ Functional Characteristics $\dagger$} & $(n=9026)$ \\
\hline $\begin{array}{l}\text { Cognitive impair- } \\
\text { ment (CPS } \geq 2 \text { ) }\end{array}$ & $26.5 \%$ & $27.3 \%$ \\
\hline Depression (DRS $\geq 3$ ) & $7.3 \%$ & $8.6 \%$ \\
\hline $\begin{array}{l}\text { ADL dependency } \\
\text { (ADL hierarchy } \geq 2)\end{array}$ & $17.0 \%$ & $22.0 \%$ \\
\hline $\begin{array}{l}\text { IADL dependency } \\
\text { (IADL involvement } \\
\geq 2 \text { ) }\end{array}$ & $81.5 \%$ & $87.7 \%$ \\
\hline \multicolumn{3}{|c|}{ Medication Usage in Prior 7 Days } \\
\hline$\geq 1$ medication & $91.8 \%$ & $96.5 \%$ \\
\hline$\geq 6$ medications & $53.8 \%$ & $61.0 \%$ \\
\hline$\geq 9$ medications & $26.0 \%$ & $32.9 \%$ \\
\hline $\begin{array}{l}\text { Psychotropic drug } \\
\text { use }\end{array}$ & $30.3 \%$ & $39.6 \%$ \\
\hline \multicolumn{3}{|c|}{$\begin{array}{l}\text { *The description of the home care population is drawn from a report based on all } \\
\text { long-term clients opened as of December 31, 2004. For age, gender and geographic } \\
\text { distribution, all clients were included ( } n=10,991 \text { ). For functional and medication use } \\
\text { characteristics, only clients with Minimum Data Set for Home Care (MDS-HC) assess- } \\
\text { ments completed in the previous year were included ( } n=9026 \text { ). } \\
\text { †Functional characteristics, based on MDS-HC, include: CPS (Cognitive Performance } \\
\text { Scale), in which a score of } 2 \text { or more indicates some level of impairment (Morris et } \\
\text { al. 1994); DRS (Depression Rating Scale), in which a score of } 3 \text { or more indicates } \\
\text { potential depression (Burrows et al. 2000); ADL Hierarchy, in which a score of } 2 \text { or } \\
\text { more indicates assistance in any of eating, toilet use, locomotion and eating (Morris } \\
\text { et al. 1999); and IADL involvement, in which a score of } 2 \text { or more indicates that at } \\
\text { least one of ordinary housework, meal preparation and/or phone use was performed } \\
\text { by someone else (Landi et al. 2000). } \\
\text { fMedications in past } 7 \text { days include both prescription and over-the-counter drugs; } \\
\text { psychotropic drug use includes any of antipsychotics/neuroleptics, anxiolytics, antide- } \\
\text { pressants, hypnotics. }\end{array}$} \\
\hline
\end{tabular}

"fall" and finding "will call client in the fall") or if the potential event clearly occurred before or after the study period (e.g., client had a fall before intake).

The remaining 215 clients ( $53.8 \%$ of sample) were forwarded for a full chart review to determine if an AE had actually 
Figure 2. Results of electronic screening methods

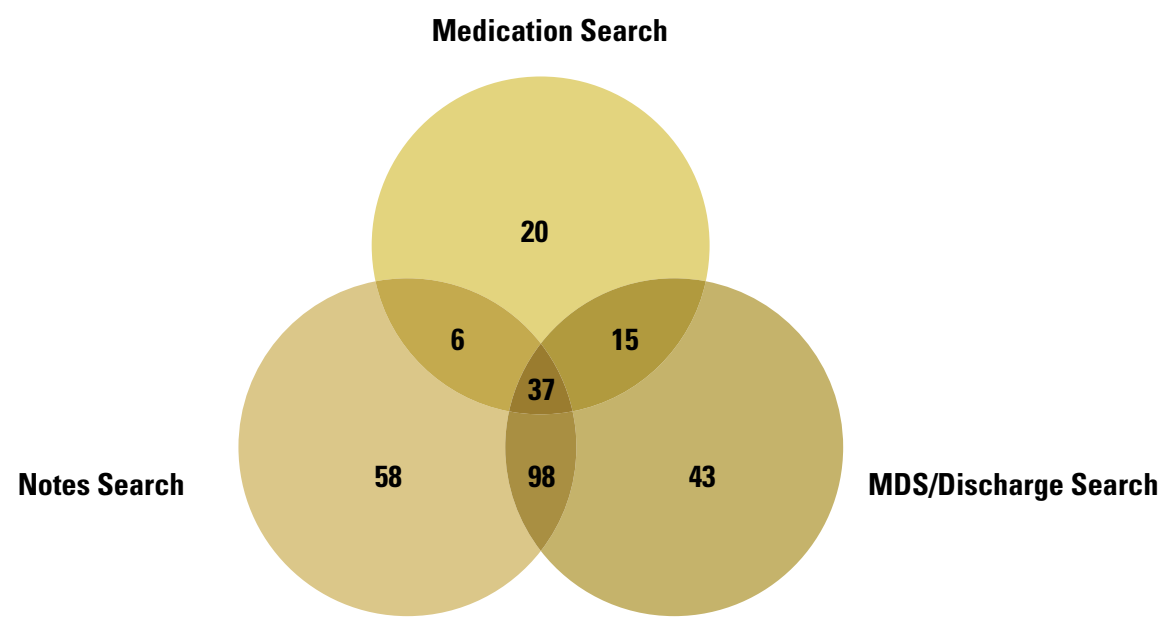

*Total of 277 clients identified in Venn diagram. The additional two clients that make up the total 279 identified by screening were identified exclusively by an occurrence report; of the seven clients identified by occurrence reports, five were also identified by an electronic screening technique.

occurred. Of these clients, 22 were found to have experienced at least one $\mathrm{AE}$, indicating a $5.5 \%$ annual incidence of AEs among the sample (95\% CI 3.3\%-7.7\%). Twenty-six AEs were found among these 22 clients, with three clients having had multiple events.

Injurious falls accounted for nearly half (46.2\%) of the 26 AEs, followed by medication-related events $(23.1 \%)$, noninjurious falls (15.4\%), pressure ulcers (3.8\%), mental harm/ injury (3.8\%) and other (7.7\%). Most of the AEs (69.3\%) resulted in temporary harm or injury to the client. One of the 26 events caused permanent harm. For the remaining events, the level of harm was listed as otherwise unneeded hospitalization $(15.4 \%)$, premature nursing home placement $(3.8 \%)$ and other $(7.7 \%)$. No events were found that resulted in a death.

Chart reviewers also identified providers that were associated with the AE. Reviewers identified multiple contributing providers in $46.2 \%$ of events. Fifty percent of AEs were associated with home care, and all but one of these were related to case management issues rather than to direct service delivery. Informal caregivers were identified as contributing to $42.3 \%$ of AEs, and clients themselves in $30.8 \%$ of events. Other healthcare providers, identified by chart reviewers as family physicians and hospitals, were associated with $42.3 \%$ of the AEs.

Chart reviewers also judged whether the AE could have been prevented or ameliorated. Of the 26 events identified, $42.6 \%$ were rated as ameliorable and $26.9 \%$ as preventable. Among the 22 clients who experienced at least one $\mathrm{AE}, 45.5 \%$ had an $\mathrm{AE}$ that was ameliorable and $27.3 \%$ had an $\mathrm{AE}$ that was preventable. This means that $4.0 \%$ of the sample experienced an $\mathrm{AE}$ that was either preventable or ameliorable (95\% CI 2.1\%-5.9\%).

Many of the reviewers' comments about preventability and ameliorability reflect "system" issues, such as practice and communication. Most comments related to the absence of or delay in referrals for occupational or physical therapy that might have prevented or ameliorated falls. The involvement, or noninvolvement, of other providers (e.g., physicians, hospitals) was also noted as a contributing cause, with specific examples related to communication challenges between providers, premature hospital discharge and delays in referrals.

Further analysis by contributing providers found that nearly all of the events associated with home care were rated as preventable or ameliorable (94.5\%). For AEs for which other providers were responsible, reviewers rated only $36.4 \%$ as ameliorable or preventable. Nearly all (92.6\%) of the home care AEs were falls while adverse drug events (ADEs) accounted for just over half (54.3\%) of the AEs for which other providers were responsible. Table 4 provides a description of the types, preventability and ameliorability of AEs by responsible providers.

One final aspect of the study, which applies only to clients 65 years and older ( $n=326$ or $81.5 \%$ of sample), identified clients taking potentially inappropriate medications. A total of 78 clients, or $23.9 \%$ of seniors in the sample $(95 \%$ CI $19.4 \%-$ $28.5 \%$ ), had taken potentially inappropriate medication. While many of these medications are listed as high-risk by the Beers 
expert panel (Beers 1997; Fick et al. 2003), very few AEs were found among clients taking these medications. Eight of these 78 clients had an AE, and two of these were classified as a drugrelated event.

\section{Discussion}

Extrapolating the results from this sample of Winnipeg home care clients suggests that 478 to 1,131 WRHA long-term home care clients likely experienced an AE in 2004. Of these events 304 to 866 were potentially preventable or ameliorable.

The annual incidence of home care AEs is lower than the rate of 7.5\% found in Canadian hospitals (Baker et al. 2004), but higher than the rates of 2.9\% and 3.7\% found in American hospitals (Brennan et al. 1991; Thomas et al. 2000). It is considerably lower than rates of $19 \%$ and $23.2 \%$ found among hospital patients following discharge (Forster et al. 2003; Forster et al. 2004). The preventability and ameliorability ratings for this study, $26.9 \%$ and $42.3 \%$ respectively, are slightly higher than those found in post-discharge studies by Forster et al. (2003; 2004).

Home care was judged to be a contributing provider in half of all AEs, while other providers (family physicians and hospitals), informal caregivers and clients themselves were identified as contributing to many events. This confirms findings from both the literature and consultation sessions about the fragmentation of care provision for home care clients. Client choices and family care can create difficult situations that escalate client risk and possibly result in an AE. Clearly, the quality of care for clients relies not only on quality home care services, but also on quality care from other healthcare providers and informal caregivers, as well as educated client and family choices about risk and care.

As this was one of the first studies to examine AEs in home care, it provides an important starting point for understanding safety issues in this setting. The approach used to adapt hospitalcentred patient safety concepts and methods could prove useful not only to other home care programs, but also in other care settings (e.g., mental health, public health, primary care and long-term care), which have also been largely overlooked in patient safety research.

These findings offer important information about "client safety" and have direct implications for home care quality improvement that might help to reduce the incidence of AEs. Results suggest that client safety may be improved if two broad issues were addressed. First, nearly all of the home care associated AEs were falls, and the most common method suggested by reviewers to prevent or ameliorate falls was a referral for occupational or physical therapy. A falls prevention program and a review of the use of therapy services might be effective strategies and have the largest impact on client safety.

The second issue relates to the fragmentation of care provision for clients. Stronger communication and collaboration between home care and other providers might help to reduce the number of events attributed to these providers. Furthermore, additional client and family education might help to prevent or ameliorate AEs.

Table 4. Adverse events by contributing provider

\begin{tabular}{|c|c|c|c|c|c|c|}
\hline & & \multicolumn{2}{|c|}{ Home Care Providers } & \multicolumn{3}{|c|}{ Non-Home Care Providers } \\
\hline & $\begin{array}{l}\text { All Adverse Events } \\
(\%)(n=26)\end{array}$ & $\begin{array}{l}\text { Direct Service } \\
\text { Staff }(\%)(n=1)\end{array}$ & $\begin{array}{l}\text { Coordinators } \\
(\%)(n=12)\end{array}$ & $\begin{array}{l}\text { Clients (\%) } \\
(n=7)\end{array}$ & $\begin{array}{l}\text { Caregivers (\%) } \\
\qquad(n=11)\end{array}$ & $\begin{array}{l}\text { Other Providers }(\%) \\
\qquad(n=11)\end{array}$ \\
\hline \multicolumn{7}{|c|}{ Type of Adverse Event } \\
\hline Fall, injurious & 46.2 & 0.0 & 66.7 & 14.3 & 54.5 & 27.3 \\
\hline Fall, non-injurious & 15.4 & 100.0 & 25.0 & 28.6 & 18.2 & 9.1 \\
\hline Pressure ulcer & 3.8 & 0.0 & 0.0 & 0.0 & 0.0 & 9.1 \\
\hline Adverse drug event & 23.1 & 0.0 & 0.0 & 28.6 & 9.1 & 54.6 \\
\hline Mental harm/injury & 3.8 & 0.0 & 8.1 & 14.3 & 9.1 & 0.0 \\
\hline Other & 7.7 & 0.0 & 0.0 & 14.3 & 9.1 & 0.0 \\
\hline \multicolumn{7}{|c|}{ Preventability and Ameliorability } \\
\hline Ameliorable & 42.3 & 0.0 & 83.3 & 42.9 & 45.5 & 18.2 \\
\hline Preventable & 26.9 & 100.0 & 8.3 & 0.0 & 36.4 & 18.2 \\
\hline Neither & 30.8 & 0.0 & 8.3 & 57.1 & 18.2 & 63.6 \\
\hline
\end{tabular}




\section{Study Limitations}

Retrospective chart review can have several limitations, including interrater reliability (agreement between chart reviewers), incomplete documentation and loss of context, and reviewer bias (Anderson 1996; Baker 2004; Birnbaum and Scheckler 2002; Localio et al. 1996; Thomas et al. 2002; Thomas and Peterson 2003). To address interrater reliability, a subsample of $18.1 \%(\mathrm{n}=39)$ of screenedin clients received two independent reviews. Reviewers agreed $87.2 \%$ of the time that an $\mathrm{AE}$ had occurred; the Kappa statistic for judging this variable was 0.65 , described as substantial (Landis and Koch 1977). Only those units in the subsample identified as having an AE were included for comparing ratings of preventability and ameliorability. For this small number of clients $(n=7)$, reviewers agreed on all cases, a Kappa score of 1.00 , or perfect agreement. Incomplete documentation and loss of context are difficult to assess in a retrospective setting. In this study, reviewers rated the adequacy of each reviewed record and deemed nearly all charts $(96.8 \%)$ as adequate or having only slight deficiencies.

It remains possible that additional AEs occurred but were not documented in the home care file. Specifically, events caused by other healthcare providers, informal caregivers and clients may not have been recorded and, consequently, may have been missed in this study. The rate of ADEs found in the sample $(1.0 \%)$ was lower than the $20.1 \%$ incidence of ADEs found among home care clients post-hospital discharge (Gray et al. 1999). This difference raises concerns that additional ADEs may have been missed.

Additional research may help to address these limitations. A more comprehensive approach for identifying ADEs is needed - perhaps linking client data with other healthcare databases, such as hospital abstracts and physician billing information. Also, this study was limited to Winnipeg clients; research in other jurisdictions would allow for a broader understanding of client safety in home care.

To view Appendix see http://www.longwoods.com/product. php?productid $=18377$ o $c$ at $=452$

\section{About the Author}

Keir G. Johnson, MPA, is Program Evaluation Specialist, Research and Evaluation Unit, Division of Research and Applied Learning, Winnipeg Regional Health Authority. His research interests include patient safety in community care.

\section{$\checkmark$ Practice Improves Safety}

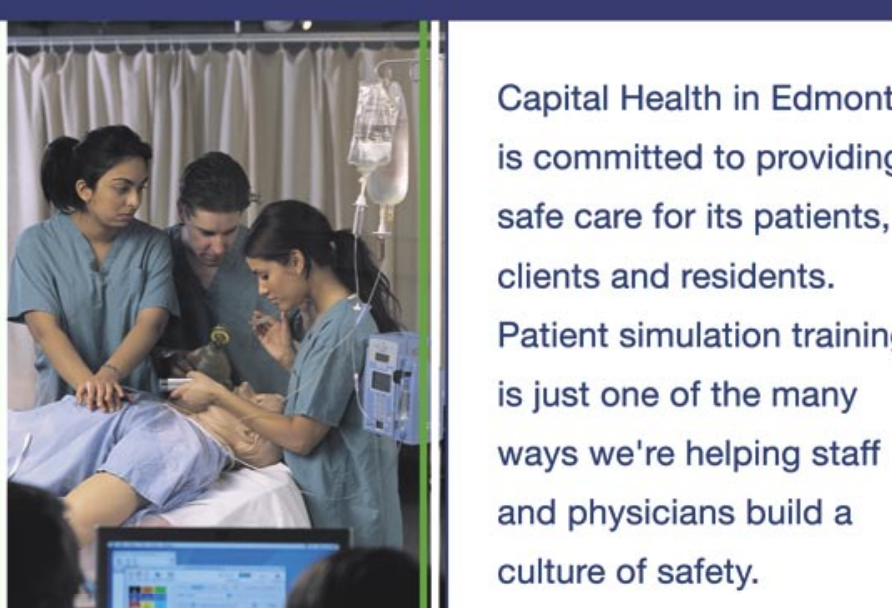

www.capitalhealth.ca

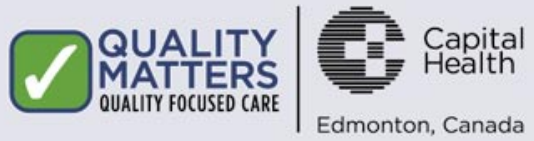

Please direct correspondence to: Keir Johnson, Winnipeg Regional Health Authority, 1800-155 Carlton Street, Winnipeg, MB R3C 4Y1; Tel: (204) 926-8065; Fax: (204) 947-9970; E-mail: kjohnson1@wrha.mb.ca.

\section{Acknowledgments}

The author would like to acknowledge Dr. Paul Thomas, Professor at the University of Manitoba and Chair of the Manitoba Institute for Patient Safety, for his ongoing support and advice for this study. Also, this study would not have been possible without the volunteered chart review time of Lynne Anderson, Shirley Labman, Leslie Orlikow and Janis Wisher.

\section{References}

Anderson, R. 1996. "An Epidemic of Medical Malpractice? A Commentary on the Harvard Medical Practice Study." Civil Justice Memo (Center for Legal Policy at the Manhattan Institute) 27 (July 1996). Retrieved August 17, 2006. <http://www.manhattan-institute. org/html/cjm_27.htm>

Baker, G. Ross. 2004. "Commentary: Harvard Medical Practice Review." Quality and Safety in Health Care 13: 151-52.

Baker, G.R., P.G. Norton, V. Flintoft, R. Blais, A. Brown, J. Cox, E. Etchells, W.A. Ghali, P. Hébert, S.R. Majumdar, M. O’Beirne, L. Palacios-Derflingher, R.J. Reid, S. Sheps and R. Tamblyn. 2004. 
"The Canadian Adverse Event Study: The Incidence of Adverse Events among Hospital Patients in Canada." Canadian Medical Association Journal 170(11): 1678-86.

Beers, M.H. 1997. "Explicit Criteria for Determining Potentially Inappropriate Medication Use by the Elderly." Archive of Internal Medicine 157(14): 1531-36.

Birnbaum, D. and William Scheckler. 2002. "Beware of the Patient Safety Juggernauts." British Journal of Clinical Governance 7(4): 28285.

Brennan, T.A., L.L. Leape, N.M. Laird, L. Hebert, A.R. Localio, A.G. Lawthers, J.P. Newhouse, P.C. Weiler, and H.H. Hiatt. 1991. "Incidence of Adverse Events and Negligence in Hospitalized Patients: Results of the Harvard Medical Practice Study I.” New England Journal of Medicine 324(6): 370-77.

Bruno, L. and J. Ahrens. 2005. "Examining 'Operational Failures' to Reduce Home Care Errors." Caring 24(2): 34-35, 37, 39.

Burrows, A.B., J.N. Morris, S.E. Simon, J.P. Hirdes and C.H. Phillips. 2000. "Development of a Minimum Data Set-Based Depression Rating Scale for Use in Nursing Homes." Age and Ageing 29(2): 165-72.

Ellenbeker, C.H., S.C. Frazier and S. Verney. 2004. "Nurses' Observations and Experiences of Problems and Adverse Effects of Medication Management in Home Care." Geriatric Nursing 25(3): 164-70.

Fialová, D., E. Topinková, G. Gambassi, H. Finne-Soveri, P.V. Jónsson, I. Carpenter, M. Schroll, G. Onder, L. Wergeland Sørbye, C. Wagner, J. Reissigová and R. Bernabei. 2005. "Potentially Inappropriate Medication Use among Elderly Home Care Patients in Europe." Journal of the American Medical Association 293(11): 1348-58.

Fick, D.M., J.W. Cooper, W.E. Wade, J.L. Waller, J.R. Maclean and M.H. Beers. 2003. "Updating the Beers Criteria for Potentially Inappropriate Medication Use in Older Adults." Archive of Internal Medicine 163(22): 2716-24.

Forster, A.J., J. Andrade and C. van Walraven. 2005. "Validation of a Discharge Summary Term Search Method to Detect Adverse Events." Journal of the American Medical Informatics Association 12(2): 200206.

Forster, A.J., H.D. Clark, A. Menard, N. Dupuis, R. Chernish, N. Chandok, A. Khan and C. van Walraven. 2004. "Adverse Events among Medical Patients after Discharge from Hospital." Canadian Medical Association Journal 170(3): 345-49.

Forster, A.J., H.J. Murff, J.F. Peterson, T.K. Gandhi and D.W. Bates. 2003. "The Incidence and Severity of Adverse Events Affecting Patients after Discharge from the Hospital." Annals of Internal Medicine 138(3): 161-67.

Golden, A.G., R.A. Preston, S.D. Barnett, M. Llorente, K. Hamdan and M.A Silverman. 1999. "Inappropriate Medication Prescribing in Homebound Older Adults." Journal of the American Geriatrics Society 47(8): 948-53.

Gray, S.L., J.E. Mahoney and D.K. Blough. 1999. "Adverse Drug Events in Elderly Patients Receiving Home Health Services following Hospital Discharge." Annals of Pharmacotherapy 33(11): 1147-53.

Landi, F., E. Tua, G. Onder, B. Carrara, A. Sgadari, C. Rinaldi, G. Gambassi, F. Lattanzio and R. Bernabei. 2000. "Minimum Data Set for Home Care: A Valid Instrument to Assess Frail Older People Living in the Community." Medical Care 38(12): 1184-90.

Landis, J.R. and G.G. Koch. 1977. "The Measurement of Observer Agreement for Categorical Data.” Biometrics 33(1): 159-74.
Localio, A.R., S.L. Weaver, J.R. Landis, A.G. Lawthers, T.A. Brenhan, L. Hebert and T.J. Sharp. 1996. "Identifying Adverse Events Caused by Medical Error: Degree of Physician Agreement in a Retrospective Chart Review." Annals of Internal Medicine 125(6): 457-64.

Madigan, E.A., D. Schott and C.R. Matthews. 2001. "Rehospitalization among Home Healthcare Patients: Results of a Prospective Study." Home Healthcare Nurse 19(5): 298-305.

Madigan, E.A. and S. Tullai-McGuinness. 2004. "An Examination of the Most Frequent Adverse Events in Home Care Agencies." Home Healthcare Nurse 22(4): 256-62.

McLeod, P.J., A.R. Huang, R.M. Tamblyn and D.C. Gayton. 1997. "Defining Inappropriate Practices in Prescribing for Elderly People: A National Consensus Panel." Canadian Medical Association Journal 156(3): 385-91.

Meredith, S., P.H. Feldman, D. Frey, K. Hall, K. Arnold, N.J. Brown and W.A. Ray. 2001. "Possible Medication Errors in Home Healthcare Patients." Journal of the American Geriatrics Society 49(6): 719-24.

Micromedex ${ }^{\circledR}$ Healthcare Series. 2005 (edition expires 2005). Greenwood Village, CO: Thomson Micromedex. Available online at Lyman Maynard Stowe Library at the University of Connecticut Health Center site. Retrieved August 17, 2006. <http://libraryweb. uchc.edu/update/archives/2005/08/micromedex_new.html>

Morris, J.N., B.E. Fries, D.R. Mehr, C. Hawes, C. Phillips, V. Mor and L.A. Lipsitz. 1994. "The MDS Cognitive Performance Scale." Journal of Gerontology 49(4): 174-82.

Morris, J.N., B.E. Fries and S.A. Morris. 1999. "Scaling ADLs within the MDS." Journals of Gerontology Series A: Biological Sciences and Medical Sciences 54(11): M546-M553.

Morris, J.N., B.E. Fries, K. Steel, N. Ikegami, R. Bernabei, G.I. Carpenter, R. Gilgen, J.P. Hirdes and E. Topinkova. 1997. "Comprehensive Clinical Assessment in Community Setting: Applicability of the MDS-HC." Journal of the American Geriatrics Society 45(8): 1017-24.

Murff, H.J., A.J. Forster, J.F. Peterson, J.M. Fiskio, H.L. Heiman and D.W. Bates. 2003. "Electronically Screening Discharge Summaries for Adverse Medical Events." Journal of the American Medical Informatics Association 10(4): 339-50.

Roos, N., L. Stranc, S. Peterson, L. Mitchell, B. Bogdanovic and E. Shapiro. 2001. A Look at Home Care in Manitoba. Winnipeg, MB: Manitoba Centre for Health Policy and Evaluation. Retrieved September 25, 2004. <https://www.umanitoba.ca/centres/mchp/ reports/pdfs/homecare.pdf>

Thomas, E.J., S.R. Lipsitz, D.M. Studdert and T.A. Brennan. 2002. "Reliability of Medical Record Review for Estimating Adverse Event Rates." Annals of Internal Medicine 136(11): 812-16.

Thomas, E.J. and L.A. Petersen. 2003. "Measuring Errors and Adverse Events in Health Care." Journal of General Internal Medicine 18(1): 261-67.

Thomas, E.J., D.M. Studdert, H.R. Burstin, E.J. Orav, T. Zeena, E.J. Williams, K.M. Howard, P.C. Weiler and T.A. Brennan. 2000. "Incidence and Types of Adverse Events and Negligent Care in Utah and Colorado." Medical Care 38(3): 261-71.

Winnipeg Regional Health Authority. 2005. Home Care Report on Community Areas: RAI-HC Data, 2004. Winnipeg, MB: Winnipeg Regional Health Authority.

Woloshynowych, M., G. Neale and C. Vincent. 2003. "Case Record Review of Adverse Events: A New Approach." Quality and Safety in Health Care 12(6): 411-15. 